\title{
Universal LC Method for a Determination of Fourteen Cationic Surfactants Widely Used in Surfactant Industry
}

\author{
Ho Ryul Ryu, Hong Soon Park, and Choong Kyun Rhee \\ Department of Chemistry, Chungnam National Lniversity, Daejeon 305-764, Korea. "E-mail: ckrheeachu.ac.kr \\ Received October 11, 2006
}

\begin{abstract}
This work presents a universal and simple LC method with evaporative light scattering detection (ELSD), for a determination of fourteen widely used cationic surfactants in industry (DDTM. DDBA. TDTM. TDBA. MTOA. THA. HDTM. ODTM. TOA. DLDM. DMDM. DPDM. IM and DSDM). The LC system employs a reverse phase LC method utilizing a single J'sphere ODS $(250 \times 4.6 \mathrm{~mm} .4 \mu \mathrm{m})$ column and a methanol-water containing $0.15 \%$ TFA elıent system. The ELSD responds to all the surfactants of interest: the linear working ranges in the $\log -\log$ plots are $2-2800 \mu \mathrm{g} \mathrm{mL}^{-1}$ with precision less than $5 \%$. and the detection limits are in the concentration range of $1.5-7.5 \mu \mathrm{g} \mathrm{mL}^{-1}$. The application of the analytical procedure to two household products without pretreatment indicates that the presented chromatographic method would be universal and simple in the analy'sis of cationic surfactants in commercial products.
\end{abstract}

Key Words : Cationic surfactant. LC. ELSD

\section{Introduction}

Surfactants of various types are very important ingredients in conmercial products for ordinary life and industry. The target of surfactant analysis would be twofold: quality assurance for commercial products and pollution control in environment. ${ }^{l}$ In assay of surfactant products. the analytical methods should be able to identify surfactants of various types universally in a simple way. Furthermore. the detection limit in the range of a few $\mu \mathrm{g} \mathrm{mL}^{-1}$ would be good enough. because the concentrations of sample surfactant solution in industrial products are generally in the order of a few tens $\mu \mathrm{g} \mathrm{mL}^{-1}$. In conjunction with pollution control. on the other hand. the tecluniques to identify and quantify the surfactants released from the surfactant products to the environments would require much lower detection limits at the level of trace analy sis. ${ }^{23}$ The constraint of extremely low detection limit is due to huge dilution and biodegradation of the released surfactants in environments. It is known that the concentration of the surfactants in environment is normally in the order of a few $\mathrm{ng} \mathrm{mL^{-1 }}$. Therefore. the requisites for an analytical method of surfactants depend on the specific purposes.

Several analytical methods have been developed for the analysis of surfactants of various types. Concerning the quality control in the surfactant industry. however the developed tecluniques do not meet the general requisites mentioned as above. Two phase titration for quaternary ammonium compounds (QACs). although simple. is not able to distinguish homologues of a surfactant.$^{+-6}$ Capillary electrophoresis. in spite of high separation efficiency and speed. needs tremendous attentions to experimental variables for reproducibility. ${ }^{7-10}$ Gas chromatography is always associated with problems such as pyrolysis and demethylation. ${ }^{\text {Il-13 }}$ On the other hand liquid chromatography with mass spectrometric detection (LC-MS) may be proper for the analysis of QACs in environmental sample. ${ }^{14.15}$ but not good for industrial application due to complexity in operation and high equipment cost

For the purpose of quality assurance for inspection of industrial products (possibly for quality control in industry as well), we have reported recently simple liquid chromatographic methods conbined with evaporative light scattering detection (ELSD). The major achievement of our previous works is development of chromatographic conditions to separate simultaneously surfactants nuixtures (nonionic-anionic and cationic-nonionic-amphoteric) with ODS column and methanol/water. ${ }^{16.17}$ In addition, the employment of an ELSD eliminates the complication in detection of the various surfactants such as chemical derivatization prior to sample injection. so that universality and simplicity in detection of the separated surfactants have been established. Furthermore. the detection limits measured for each surfactant are good for industrial application.

An analytical method specifically focused on the cationic surfactants commonly used in industry. 14 cationic surfactants (quaternary amimonium compounds (QACs), benzylalkyldimethylammonium salts (BZAs) and imidazolinium salts (IMs)). would be challenging for quality assurance and/ or quality control. A reason for the particular need is that many cationic surfactants are added into various industrial products such as hair rinse fabric softener. corrosion inhibitors and antimicrobial agents. The method described in the previous report. ${ }^{17}$ does not cover all the previously mentioned cationic surfactants. although good for a few cationic surfactants. In this work, therefore. we aimed a universal LC method to quantify a mixture of the 14 cationic surfactants commonly used in industry at the level of quality assurance.

\section{Experimental Section}

Reagents and chemicals. The studied surfactants were as 
follows: Dodecyltrimethylamınoniuın bromide (DD']M, Sigına Chemical, USA), Tetradecyltrimethylammonium bromide (ID'M, Fluka, Switzerland). Hexadecyltrimethylammonium bromide (HDTM, Fluka, USA), Octadecyltrimethylamınonium bromide (OD'M, Sigma Chemical, USA). Dilauryldimethylanunonium bromide (DLDM, Tokyo Kasei Kogyo Ltd.. Japan). Dimyristyldimethylammonium bromide (DMDM, Tokyo Kasei Kogyo Ltd., Japan). Dipalmityldimethylammonium bromide (Dl'DM, Tokyo Kasei Kogyo Ltd., Japan). Distearyldimethylammonium bromide (DSDM, 'Tokyo Kasei Kogyo Ltd., Japan). Dodecyldimethylbenzylamınonium chloride (DDBA. Sigma Chemical, USA), Tetradecyldimethylbenzylamınonium chloride (IDBA, Sigma Chemical, USA), Methyltrioctylammonium bromide (M'IOA, Sigma Chemical, USA), Tetraheptylammonium bromide (IHA, Sigma Chemical, USA). Tetraoctylammonium bromide (IOA, Sigma Chemical, USA) and Imidazolinium (IM, Sunjin Chemical Ltd., South Korea). All the surfactants were used as received.

Chromatographic conditions. The LC apparatus (Hewlett Packard 1050 Chemstation. USA) employed an YMC J'sphere C18 $(250 \times 4.6 \mathrm{~mm}, 4, \mu \mathrm{m})$ column and an eluent system consisting of methanol and water containing $0.15 \%$ trifluoroacetic acid (THA). Specifically, T'HA (HPLC grade, Fisher Scientific Co., USA) was added to water (>18 M $\Omega$ $\mathrm{cm}^{-1}$. Mili-Q, USA) and methanol (HPLC grade, Fisher Scientific Co., USA) to be $0.15 \%$ by volume. The initial eluent solution was a mixture of $75 \%$ methanol- $25 \%$ water, and the injected amount of a sample was a $20 \mu \mathrm{L}$. After the injection, the content of methanol in the eluent was increased linearly from $75 \%$ to $100 \%$ for $50 \mathrm{~min}$ and held for $7 \mathrm{~min}$. Following the advent of the last peak at $52 \mathrm{~min}$, the eluent condition was maintained for cleaning up the column for 5 min more. Then, the initial eluent condition was resumed and held for $7 \mathrm{~min}$ to re-equilibrate the column prior to the subsequent injection. The flow rate of the eluent solution was $0.8 \mathrm{~mL} \mathrm{~min}^{-1}$ throughout the whole work.

The detection was performed utilizing an ELSD (Alltech 500 , U.S.A) whose signal was hooked up to a personal computer. The ELSD was warmed up for $20 \mathrm{~min}$ prior to each run, and operated at $95^{\circ} \mathrm{C}$. The optimum flow rate of a nebulizing gas $\left(\mathrm{N}_{2}, 99.99 \%\right.$ ) was $2.85 \mathrm{~L} \mathrm{~min}^{-1}$.

The operating conditions for gradient elution and detection were optimized as much as possible. It is worthy to address, furthermore, that in the preliminary stage of the optimization process, various eluents (water, methanol and acetonitrile) and stationary phases (C4 and $\mathrm{C} 8$ ) were examined also.

Preparation of standard and sample solutions. Stock solutions of each surfactant under study were prepared in aqueous methanolic solution (methanol $80 \%$, by volume), and the working solutions for calibration were implemented by successive dilutions of the stock solutions with the solvent. The upper concentrations of the working solutions of each surfactant were chosen, based on the solubility limit and the tolerance in column loading.

Sample solutions were prepared by dissolving two com- mercial products ( $1.0 \mathrm{~g}$ each of a hair rinse and a fabric softener) in $25 \mathrm{~mL}$ of the aqueous methanolic solution with a mild stirring. After keeping the solutions calm for $3 \mathrm{hr}$, only the clear upper layers of the sample solutions were allowed to pass through membrane filters. Without any further treatment, the filtered solutions were injected into the $\mathrm{LC}$. column. A fairly good reproducibility associated with the commercial products (see [able 2) indicates that the ingredients in the sample solutions did not cause any damage to the column. For spike tests, the pre-determined amounts of the corresponding standards were added to the commercial products. After homogenizing the mixture, the spike testing solutions were prepared through the identical processes as above.

Peak identification. The surfactants separated with the LC column were identified using FAB-MS (JMS-AX 505H Mass Spectrometer, JEOL, Japan).

\section{Results and Discussion}

Simultaneous separation. A chromatogram of a mixture of the studied fourteen cationic surfactants is shown in Figure I. All of the cationic surfactants were separated well enough within $52 \mathrm{~min}$ as tabulated in lable $\mathrm{l}$, and the relative standard deviations (RSD) of the observed retention times were less than $3 \%$. In each family of $\mathrm{QAC}, \mathrm{B} \angle \mathrm{A}$ and IM, the observed retention time became larger as the number of carbon increased as anticipated with the employed reverse phase LC column. On the other hand, MIOA (tri- $\mathrm{C}_{8}$ ) and [HA (tetra- $\mathrm{C}_{7}$ ) are worthy to address in detail. Although the retention times of the two compounds (lable l) differed only by $0.1 \mathrm{~min}$, their separated peaks were quite discernible as shown in the inset of Figure 1, when their concentrations were less than $200 \mu \mathrm{g} \mathrm{mL}$.

It is worthy to compare specifically the chromatographic condition in the work with that in our previous report. ${ }^{17}$ The elution gradient program in this work is quite different from that in the previous work: in this work, the content of

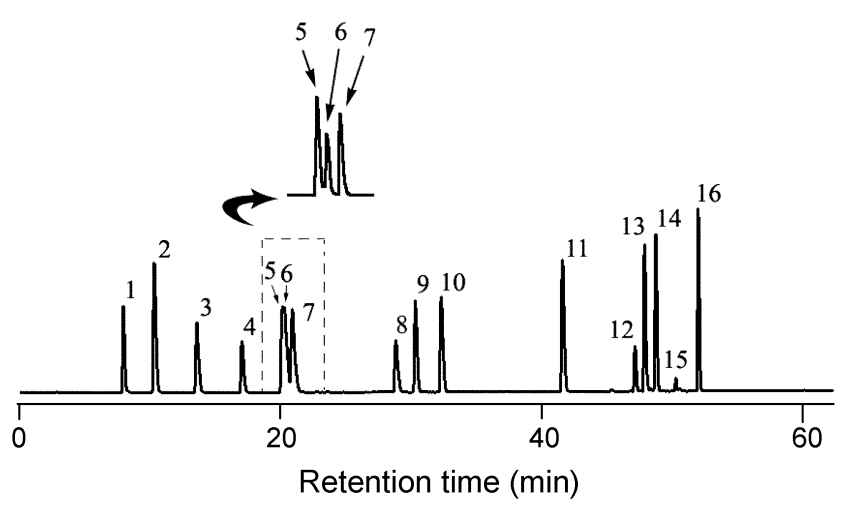

Figure 1. A chromatogram of a mixture of the fourten cationic surlactants; 1: DDTM. 2: DDBA. 3: TDTM. 4: TDBA. 5: MTOA. 6: IHA . 7: IIITM 8: OIIM. 9: IOA I0: IIIIMM 11: IDMIDM. 12: IM (C34). 13: DPLD. I4: IM (C36. dominant). 15: IM (C38). 16: DSDM. The inserted chromatogram was obtained for the surlactants of interested in low concentrations (see the text). 
methanol was linearly increased from $75 \%$ to $100 \%$ for 52 min, while in our previous work, the methanol content was held at $70 \%$ for $3 \mathrm{~min}$, linearly increased to $95 \%$ for $42 \mathrm{~min}$ and then held more for $4 \mathrm{~min}$. Because only a separation of cationic surfactants was focused in this work (recall that the previous method $^{17}$ dealt with a separation of a mixture of several cationic, nonionic and amphoteric surfactants), the employed gradient program was efticient enough in separation of the studied cationic surfactants. For example, the four surfactants (DD'M, DDBA, TD'M and TDBA) were clearly separated in the early stage under the condition in this work, while they were eluted into insufficiently separated peaks under the condition in the previous work. ${ }^{17}$ Another example is that a minor homologue of IM (C38), not recognizable in the previous work, was clearly observed. Furthermore, the retention time of DLDM reduced from 37 $\mathrm{min}$ in the previous work to $32 \mathrm{~min}$ in this work. On the other hand, the concentration of JFA $(0.15 \%)$. lower than that in the previous work $(0.20 \%)$, resulted in sharpening up the chromatographic peaks of the studied surfactants to increase the resolutions. "The described improvements are the points distinguishing this work from our previous works. ${ }^{16.17}$

Detection. The peak area measured with ELSD, $\alpha$, is not linear to the injected mass, $m^{18.19}$ For quantitative purposes. therefore, a log-log plot is generally used for each surfactant as follows:

$$
\ln a-E \ln m \mid \ln K
$$

where $E$ and $K$ are constants specific for each surfactant. From the log-log plots of the studied surfactants, the detec-

Table I. Retention times. detection limits. working ranges and loglog slopes of the sludied surlactants

\begin{tabular}{|c|c|c|c|c|c|}
\hline $\begin{array}{l}\text { peak } \\
\text { number }\end{array}$ & surfactant & $\begin{array}{l}\text { retention } \\
\text { time* } \\
(\min -\mathrm{SD})\end{array}$ & $\begin{array}{c}\text { detection } \\
\text { limit }{ }^{*} \\
\left(\mu g \mathrm{~mL}^{\prime}\right)\end{array}$ & $\begin{array}{l}\text { working } \\
\text { range } \\
\left(\mu \mathrm{g} \mathrm{mL}^{\prime}\right)\end{array}$ & $\begin{array}{l}\text { log-log } \\
\text { slope }\end{array}$ \\
\hline 1 & DDTM & $7.87=0.03$ & 6.5 & $8-2050$ & 1.464 \\
\hline 2 & DDBA & $10.29 \pm 0.03$ & 5.5 & $8-2800$ & 1.517 \\
\hline 3 & TDTM & $13.52 \pm 0.05$ & 7.0 & $8-2010$ & 1.463 \\
\hline 4 & TDBA & $17.01 \pm 0.04$ & 7.5 & $10-1650$ & 1.567 \\
\hline 5 & MTOA & $20.11 \pm 0.05$ & 5.5 & $8-2680$ & 1.410 \\
\hline 6 & THA & $20.20 \pm 0.06$ & 6.0 & $7-2040$ & 1.531 \\
\hline 7 & HDTM & $20.83 \pm 0.06$ & 6.5 & $8-2160$ & 1.491 \\
\hline 8 & ODTM & $28.76 \pm 0.06$ & 6.0 & $7-2060$ & 1.479 \\
\hline 9 & TOA & $30.29 \pm 0.08$ & 3.0 & $5-2050$ & 1.520 \\
\hline 10 & DLDM & $32.25 \pm 0.07$ & 2.5 & $5-1950$ & 1.528 \\
\hline 11 & DMDM & $41.51 \pm 0.07$ & 2.5 & $4-2000$ & 1.524 \\
\hline 12 & $\mathrm{IM}(\mathrm{C} 34)$ & $46.92 \pm 0.09$ & - & - & - \\
\hline 13 & DPDM & $47.79 \pm 0.08$ & 2.0 & $3-1980$ & 1.517 \\
\hline 14 & $\mathrm{IM}(\mathrm{C} 36)$ & $48.71 \pm 0.09$ & 6.5 & $9-1200$ & 1.582 \\
\hline 15 & $\mathrm{IM}(\mathrm{C} 38)$ & $50.30 \pm 0.10$ & - & - & - \\
\hline 16 & DSDM & $51.93 \pm 0.09$ & 1.5 & $2-2140$ & 1.527 \\
\hline
\end{tabular}

"Retention times ( $<3 \%$ RSD) are the mean values as determined with five cxperiments for each surfactant. "Detection limits in this works were determined based on experimentally detectable signals of $3 \mathrm{SA}$ level. "Working ranges sere the linear ranges in the log-log plots determined witl seven to nine standatd concentrations. tion limit, linear working range and log-log slope of each surfactant were estimated and summarized in lable I. It should be noted that the quantitative parameters of IM were estimated from the peak of its most dominant homologue (C36). The precision of the ELSD detector, expressed with RSD after five replica experiments for each concentration, is less than $5 \%$ for all studied cationic surfactants. The observed detection limits $\left(1.5-7.5 \mu \mathrm{g} \mathrm{mL} \mathrm{mL}^{-1}\right)$ are similar to the values determined with capillary electrophoresis with direct UV detection ${ }^{8}$ and with indirect photometric detection ${ }^{20}$, and the linear concentration ranges $\left(2-2800 \mu \mathrm{g} \mathrm{mL}^{-1}\right)$ in the loglog plots are wider than those of other detection method. ${ }^{10.21}$ In addition, the ELSD worked well for the studied surfactants without chromophore (only DDBA and IDBA have chromophores.) and the gradient eluent. The results obtained so far indicate that the chromatographic conditions provide an effective separation of various cationic surfactants, and that the ELSD eliminates the complications related to detection.

Spike tests and applications. The cationic surfactants in commercial products (a hair rinse and a fabric softener) were analyzed as shown in Figure 2 . Among the peaks of the
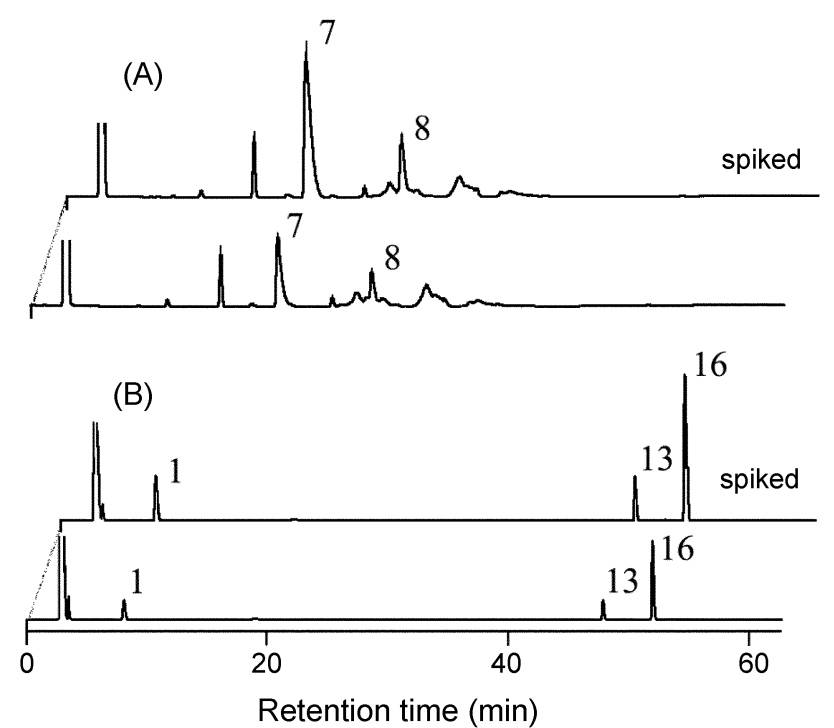

Figure 2. The chromatograms of $(A)$ hair rinse and (B) Gabric softener: (7) IIDTM. (8) ODTM. (1) DDTM. (13) DPDM. (16) DSDM. The upper ones of each chromatogram are those of the spiked samples. The unassigned peaks came from the unknown components in the sludied commercial products.

Table 2. Spike tests of two commercial products

\begin{tabular}{cccccc}
\hline \multirow{2}{*}{$\begin{array}{c}\text { commercial } \\
\text { product }\end{array}$} & surfactant & peak & \multicolumn{3}{c}{ concentration } \\
\cline { 4 - 6 } & & mumber & sample & added & total \\
\hline hair rinse & HDTM & 7 & $1.1=0.04$ & 1.0 & $2.1 \pm 0.06$ \\
& ODTM & 8 & $0.2-0.05$ & 0.2 & $0.4+0.08$ \\
fabric & DDTM & 1 & $1.5-0.07$ & 1.5 & $3.0+0.08$ \\
sottenner & DPDM & 13 & $0.7-0.05$ & 0.7 & $1.4+0.06$ \\
& DSDM & 16 & $3.0-0.05$ & 3.0 & $6.0+0.06$ \\
\hline
\end{tabular}

"The concentration values are the mean values as determined with four experiments for each commercial product. 
products on the stable base lines. the ingredient surfactants were identified and quantified as in Table 2. utilizing the figures in Table 1. Spike solutions were prepared as described in Experimental and the corresponding results indicate that there was no matrix effect at all in the analytical procedure of this work.

\section{Conclusions}

A simultaneous determination of a mixture of fourteen cationic surfactants was performed with a LC combined with ELSD. The employed chromatographic condition. YMC J'sphere CI8 reverse phase column and methanol-water gradient elution. provides a separation within approxintately 52 min. The effectiveness of this method stemmed from the employed eluent gradient in junction with ELSD to separate the 14 cationic surfactants used widely in industry. We believe that the studied method would be universally applicable to industrial products containing cationic surfactants.

\section{References}

1. Schmitt. T. M. Anatysis of Surfactants in Surfactant Science
Series: New York, U.S.A., 1992; Vol. 40

2. Ying. G. G. Enwromtent International 2006. 32.417.

3. Ding. W. H.: Liao. Y. H. Anal Chent 2001. 73. 36.

4. Llenado. R. A.: Neubecker. T. A. Anal. Chem. 1983. 55.93.

5. Sakai. T.: Ohno. N. Talanta 1986. 33, 415.

6. Yamamoto. K. And Chim. Acta 1995, 302. 75.

7. Shamsi, S. A.; Danielson. N. D. J. Chromatog: A $1996,739$. 405 .

8. Piera. E.: Erra. P.: Infante. M. R. J. Chomatogr: A 1997. 757. 275 .

9. Wang, M.: Qu. F.: Shan, X. Q.: Lin, J. M. J. Chomatogr . 2003. 989.285

10. Liu. H. Y. Ding. H. J. Chromatogs A 2004, 1025. 303.

11. Ding. W. H.: Liao. Y. H. Anal Chent 2001. 73. 36.

12. Tsai. P. C.: Ditng. W. H. J. Chomatogr A 2004. 1027. 103.

13. Grossi. K.: Vece. R J. Gas Chronatogr: 1965, 3, 170.

14. Merino. F.: Rubio. S.; Bendito. D. P. J. Chomatogr A 2003. 998. 143.

15. Ferrer. I.: Furlong. E. T. Anal Chem 2002, 74. 1275.

16. Park. H. S.: Rhee. C. K. J. Chromatogr A 2004. 10+6. 289.

17. Park. H. S.: Ryu. H. R.: Rhee. C. K. Tonlata 2006. 70.481 .

18. Bear. G. R J. Chrontatogr: 1988. 459.91.

19. Bünger. H; Kaufner, L.: Pison, U. J. Chomatogr A $2000,870$. 363.

20. Heinig. K.: Vogt, C: Werner. G. J. Chromatogr: A 1997, 781 . 17.

21. Giovannelli. D.: Abballe. F. J. Chronatogr .A 2005. 1085.86 . 